PROCEEDINGS OF THE

AMERICAN MATHEMATICAL SOCIETY

Volume 125, Number 6, June 1997, Pages 1767-1770

S $0002-9939(97) 03835-5$

\title{
A RELATION BETWEEN THE PLURICOMPLEX AND THE CLASSICAL GREEN FUNCTIONS IN THE UNIT BALL OF $\mathrm{C}^{n}$
}

\author{
MAGNUS CARLEHED
}

(Communicated by Eric Bedford)

\begin{abstract}
We give a sharp upper bound for the quotient of the pluricomplex and the classical Green functions in the unit ball of $\mathbf{C}^{n}$.
\end{abstract}

\section{INTRODUCTION}

The classical Green function for the unit ball $B=B(0,1)$ in $\mathbf{C}^{n}$ or $\mathbf{R}^{2 n}$ is

$$
G_{n}(z, w)= \begin{cases}\left.\left.|w|^{2-2 n}|z-w /| w\right|^{2}\right|^{2-2 n}-|z-w|^{2-2 n} & \text { for } w \neq 0 \\ 1-|z|^{2-2 n} & \text { for } w=0\end{cases}
$$

Here we defined it as a non-positive subharmonic function, whereas in most texts it is defined as the negative of our function (see for example $[\mathrm{H}]$, p. 77).

On the other hand, the pluricomplex Green function is $g_{n}(z, w)=\log \left|T_{w}(z)\right|$ where $T_{w}(z)$ is the Möbius transformation which maps $w$ onto the origin. To be explicit,

$$
T_{w}(z)=\frac{w-P_{w}(z)-\left(1-|w|^{2}\right)^{1 / 2} Q_{w}(z)}{1-\langle z, w\rangle}
$$

where $P_{w}$ is the orthogonal projection of $\mathbf{C}^{n}$ onto the subspace generated by $w$, and $Q_{w}$ the projection onto the orthogonal complement of that subspace $([\mathrm{K}], \mathrm{p}$. 148 and 224).

We define, for $n \geq 2$,

$$
h_{n}(z, w)=\frac{g_{n}(z, w)}{G_{n}(z, w)} .
$$

Note that $h_{n}$ is a non-negative function, which can be extended to a continuous function on $B \times B$, since

$$
\lim _{z, w \rightarrow \varsigma} h_{n}(z, w)=0 \quad \text { for all } \varsigma \in B .
$$

Received by the editors December 27, 1995.

1991 Mathematics Subject Classification. Primary 32F05; Secondary 31C05.

Key words and phrases. Classical Green function, pluricomplex Green function.

(C)1997 American Mathematical Society 


\section{The Result}

The following theorem was proved in $[\mathrm{Ca}]$.

Theorem 1. For all $z, w \in B(0,1) \subset \mathbf{C}^{n}, n \geq 2$, the inequality

$$
h_{n}(z, w)<2^{2 n-3} /(n-1)
$$

holds. Moreover, this estimate is the best possible.

Proof. In the case $w=0, h_{n}$ reduces to $\log |z| /\left(1-|z|^{2-2 n}\right)$. Let

$$
f(x)=\frac{\log x}{1-x^{2-2 n}}
$$

for all $x \in(0,1)$. Then differentiation gives

$$
f^{\prime}(x)=\frac{x^{2 n-2}+(2-2 n) \log x-1}{x^{2 n-1}\left(1-x^{2-2 n}\right)^{2}} .
$$

By differentiating the numerator of $f^{\prime}(x)$ we can easily see that it is decreasing and therefore greater than its value for $x=1$, which is 0 . Therefore $f(x)$ is increasing. Since $\lim _{x \rightarrow 1-} f(x)=1 /(2 n-2)=2^{-1} /(n-1)<2^{2 n-3} /(n-1)$, the theorem is proved in the case $w=0$.

For the rest of the proof, assume $w \neq 0$. Let $\lambda=\langle z, w\rangle /|w|^{2}$. Then $P_{w}(z)=\lambda w$ and if we let $Q_{w}(z)=z^{*}$, then $z=\lambda w+z^{*}$. Hence

$$
\begin{gathered}
g_{n}(z, w)=\frac{1}{2} \log \left|T_{w}(z)\right|^{2}= \\
=\frac{1}{2} \log \left|w-\lambda w-\left(1-|w|^{2}\right)^{1 / 2} z^{*}\right|^{2}-\frac{1}{2} \log \left|1-\left\langle\lambda w+z^{*}, w\right\rangle\right|^{2}= \\
=\frac{1}{2} \log \left(|1-\lambda|^{2}|w|^{2}+\left(1-|w|^{2}\right)\left|z^{*}\right|^{2}\right)-\left.\left.\frac{1}{2} \log |1-\lambda| w\right|^{2}\right|^{2}
\end{gathered}
$$

using the orthogonality between $z^{*}$ and $w$. By a similar argument we have

$$
\begin{gathered}
G_{n}(z, w)=\left.|w|^{2-2 n}|\lambda w-w /| w\right|^{2}+\left.z^{*}\right|^{2-2 n}-\left|(\lambda-1) w+z^{*}\right|^{2-2 n}= \\
=|w|^{2-2 n}\left(\left.\left.|\lambda w-w| w\right|^{-2}\right|^{2}+\left|z^{*}\right|^{2}\right)^{1-n}-|| \lambda-\left.1\right|^{2}|w|^{2}+\left.\left|z^{*}\right|^{2}\right|^{1-n}= \\
=\left(\left.\left.|\lambda w-w| w\right|^{-2}\right|^{2}|w|^{2}+\left|z^{*}\right|^{2}|w|^{2}\right)^{1-n}-|| \lambda-\left.1\right|^{2}|w|^{2}+\left.\left|z^{*}\right|^{2}\right|^{1-n}= \\
=\left(\left.|\lambda| w\right|^{2}-\left.1\right|^{2}+\left|z^{*}\right|^{2}|w|^{2}\right)^{1-n}-|| \lambda-\left.1\right|^{2}|w|^{2}+\left.\left|z^{*}\right|^{2}\right|^{1-n} .
\end{gathered}
$$

To simplify the notation, let $x=|w|^{2}, u=\left|z^{*}\right|^{2}|w|^{2}$. After multiplying by $G_{n}<0$ we can write the desired inequality in the following way

$$
\begin{aligned}
& \frac{1}{2} \log \left(|1-\lambda|^{2} x+(1-x) u / x\right)-\frac{1}{2} \log |1-\lambda x|^{2}> \\
& >\frac{2^{2 n-3} /(n-1)}{\left(|1-\lambda x|^{2}+u\right)^{n-1}}-\frac{2^{2 n-3} /(n-1)}{\left(|1-\lambda|^{2} x+u / x\right)^{n-1}}
\end{aligned}
$$


Since $1>|z|^{2}=|\lambda|^{2}|w|^{2}+\left|z^{*}\right|^{2}=|\lambda|^{2} x+u / x$, it suffices to show that this inequality holds for all $0<x<1,0 \leq u<x$ and $|\lambda|<\sqrt{x-u} / x$. For each $u \in[0,1)$, define

$$
f_{u, n}(t)=\log t+\frac{4^{n-1} /(n-1)}{(t+u)^{n-1}} .
$$

Our inequality now takes the form

$$
f_{u, n}\left(|1-\lambda|^{2} x+u / x-u\right)>f_{u, n}\left(|1-\lambda x|^{2}\right) \text {. }
$$

Differentiation shows that $f_{u, n}(t)$ is decreasing when $(t+u)^{n}<4^{n-1} t$. In the case $n=2$, this occurs precisely when $t \in(2-u-2 \sqrt{1-u}, 2-u+2 \sqrt{1-u})$. We claim that when $t$ belongs to this interval, then $f_{u, n}(t)$ is decreasing for all $n$. Indeed, as $(t+u)^{2}<4 t$ and $0<t<4$, we have $(t+u)^{n}=\left[(t+u)^{2}\right]^{n / 2}<(4 t)^{n / 2} \leq$ $(4 t)^{n / 2}(4 / t)^{n / 2-1}=4^{n-1} t$. Therefore it suffices to show that

$$
\begin{gathered}
2-u-2 \sqrt{1-u}<_{(1)}|1-\lambda|^{2} x+u / x-u<_{(2)} \\
<_{(2)}|1-\lambda x|^{2}<_{(3)} 2-u+2 \sqrt{1-u}
\end{gathered}
$$

for all $0<x<1,0 \leq u<x$ and $|\lambda|<\sqrt{x-u} / x$. We prove the three inequalities separately.

(1) $2-u-2 \sqrt{1-u}<|1-\lambda|^{2} x+u / x-u$

The proof is split into two cases.

Case 1. $\sqrt{x-u} / x \leq 1$.

Since $|1-\lambda| \geq 1-|\lambda|$, it is enough to prove (1) when $\lambda$ is real and non-negative. Moreover, $0 \leq \lambda<\sqrt{x-u} / x$ for such $\lambda$, so it suffices to show (1) when $\lambda=$ $\sqrt{x-u} / x$, i.e. to show that $(1-\sqrt{x-u} / x)^{2} x+u / x>2-2 \sqrt{1-u}$. Expanding the square and simplifying, we see that this is equivalent to $2 \sqrt{1-u}>1+2 \sqrt{x-u}-x$. Both sides of this inequality are non-negative, so it is enough to prove the squared inequality, namely (after simplifying) $x+3>4 \sqrt{x-u}$. It is easy to check that this is true, even for $u=0$.

Case 2. $\sqrt{x-u} / x>1$, i.e. $u<x-x^{2}$.

In this case $|1-\lambda|^{2} x$ can be zero, so we have to show the inequality without that term, i.e. we must prove that $u / x>2-2 \sqrt{1-u}$, or equivalently $u /(2-2 \sqrt{1-u})>x$. If we view the left side of this inequality as a function of $u$, we can easily see, by differentiation, that it is decreasing and therefore greater than its value in $u=x-x^{2}$ which is $\left(x-x^{2}\right) /\left(2-2 \sqrt{1-x+x^{2}}\right)$. Elementary calculation shows that this expression is greater than or equal to $x$, and (1) is proved.

(2) $|1-\lambda|^{2} x+u / x-u<|1-\lambda x|^{2}$

By using the bound $|\lambda|<\sqrt{x-u} / x$ we obtain

$$
\begin{gathered}
|1-\lambda|^{2} x+\frac{u}{x}-u<\left(1+|\lambda|^{2}-2 \operatorname{Re}(\lambda)\right) x+\left(\frac{1}{x}-1\right)\left(x-|\lambda|^{2} x^{2}\right)= \\
=1+|\lambda|^{2} x^{2}-2 \operatorname{Re}(\lambda) x=|1-\lambda x|^{2} .
\end{gathered}
$$

(3) $|1-\lambda x|^{2}<2-u+2 \sqrt{1-u}$

The same bound as used in the proof of (2) gives

$$
|1-\lambda x|^{2} \leq(1+|\lambda x|)^{2} \leq(1+\sqrt{x-u})^{2}=1+x-u+2 \sqrt{x-u}
$$


Therefore

$$
\begin{gathered}
|1-\lambda x|^{2}-(2-u+2 \sqrt{1-u}) \leq \\
\leq 1+x-u+2 \sqrt{x-u}-(2-u+2 \sqrt{1-u})= \\
=x-1+2(\sqrt{x-u}-\sqrt{1-u})<0
\end{gathered}
$$

since $x<1$. This completes the proof of (3).

It remains to show that the estimate is sharp. Let $w=(t, 0, \ldots, 0)$ and $z=$ $(-t, 0, \ldots, 0)$, where $t$ is real and positive. Then $\lambda=-1$ and $z^{*}=0$. We substitute this in the expression for $h_{n}$. Then using Taylor's formula near $t=1$ and the identity $a^{m}-b^{m}=(a-b)\left(a^{m-1}+a^{m-2} b+\ldots+b^{m-1}\right)$ we get

$$
\begin{gathered}
h_{n}(z, w)=\frac{\log (2 t)-\log \left(1+t^{2}\right)}{\left(1+t^{2}\right)^{2-2 n}-(2 t)^{2-2 n}}= \\
=-\frac{(2 t)^{2 n-2}\left(1+t^{2}\right)^{2 n-2}}{\left((2 t)^{2}\right)^{n-2}+\left((2 t)^{2}\right)^{n-3}\left(1+t^{2}\right)^{2}+\ldots+\left(\left(1+t^{2}\right)^{2}\right)^{n-2}} \times \\
\times \frac{\log 2+(t-1)-(t-1)^{2} / 2+O(t-1)^{3}-\left(\log 2+(t-1)+O(t-1)^{3}\right)}{(1-t)^{2}(1+t)^{2}}= \\
=-\frac{(2 t)^{2 n-2}\left(1+t^{2}\right)^{2 n-2}}{\left((2 t)^{2}\right)^{n-2}+\left((2 t)^{2}\right)^{n-3}\left(1+t^{2}\right)^{2}+\ldots+\left(\left(1+t^{2}\right)^{2}\right)^{n-2}} \times \\
\times\left[-\frac{1}{2}+O(t-1)\right] \frac{1}{(1+t)^{2}} \rightarrow \\
\rightarrow-\frac{2^{2 n-2} \cdot 2^{2 n-2}}{2^{2}\left[\left(2^{2}\right)^{n-2}(n-1)\right]} \cdot\left(-\frac{1}{2}\right)=\frac{2^{2 n-3}}{n-1}
\end{gathered}
$$

when $t \rightarrow 1$.

Remark. The example at the end of the proof shows that $h_{n}(z, w)$ tends to its supremum, when $z$ and $w$ approach opposite points at the boundary. Since also $h_{n}(z, w)$ has its least value 0 for $z=w$, we conjecture that

$$
\sup _{|z| \leq|w|} h_{n}(z, w)=h_{n}(-w, w)
$$

for all $w \in B$.

\section{REFERENCES}

[Ca] Carlehed, M.: Some Properties of the Pluricomplex Green Function and Potentials, Research Reports No. 14, 1995, Umeå University

[H] Helms, L.L.: Introduction to Potential Theory, Wiley-Interscience 1969 MR 41:5638

[K] Klimek, M.: Pluripotential Theory, Clarendon Press 1991 MR 93h:32021

Mid Sweden University, 83125 Östersund, Sweden

E-mail address: magnus.carlehed@ter.mh.se 